\title{
Hilbert Implication Algebra and Some Properties
}

\author{
Dejen Gerima Tefera \\ Department of Mathematics, Wollo University, College of Natural Science, Dessie, Ethiopia \\ Correspondence should be addressed to Dejen Gerima Tefera; gerima233@gmail.com
}

Received 6 April 2021; Revised 17 June 2021; Accepted 27 June 2021; Published 21 July 2021

Academic Editor: Qiankun Song

Copyright (C) 2021 Dejen Gerima Tefera. This is an open access article distributed under the Creative Commons Attribution License, which permits unrestricted use, distribution, and reproduction in any medium, provided the original work is properly cited.

\begin{abstract}
The concepts of Hilbert implication algebra and generalized Hilbert implication algebra are introduced. The comparison theorem of Hilbert implication algebra and generalized Hilbert implication algebra is proved. In addition, the idea of groupoid and commutative Hilbert implication algebras is investigated. Ideals and filters in Hilbert implication algebras are also discussed. In general, different theorems which show different properties are proved.
\end{abstract}

\section{Introduction}

Hilbert algebras are important in investigating certain algebraic logic, since they can be considered as a fragment of any propositional logic containing logical connective implications. The concept of $\mathrm{a} \otimes$-closed set and a $\otimes-$ homomorphism in lattice implication algebras and some of their properties is elaborated by Roh et al. in [1].

Commutative Hilbert algebra was initiated by Halas in [2]. The concepts of preimplication algebra and implication algebra based on orthosemilattice which generalize the concepts of implication algebra was discussed by Chajda in [3], and the notion of generalized Hilbert algebra with some other properties was investigated by Borzooei and Shohani in [4].

The notion of B-Almost distributive fuzzy lattice in terms of its principal ideal fuzzy lattice was introduced by Assaye et al. in [5]. The concepts of Pseudo - Supplemented almost distributive fuzzy Lattice with some other propertis is introduced by Gerima Tefera in [6].

In this paper, we introduced the concepts of Hilbert implication algebra and generalized Hilbert implication algebras are investigated. Furthermore, groupoids in an implication algebra, commutative properties in a Hilbert implication algebra with some other properties are introduced.

Throughout this paper, $H$ represents Hilbert implication algebra unless otherwise mentioned.

\section{Preliminaries}

Definition 1 (see [7]). An algebra $(B, \Rightarrow, 1)$ of type $(2,0)$ is called implication algebra if the following condition holds:

(1) $a \Rightarrow a=1, \forall a \in B$

(2) $a \Rightarrow 1=1, \forall a \in B$

(3) $1 \Rightarrow a=a, \forall a \in B$

(4) $a \Rightarrow(b \Rightarrow c)=b \Rightarrow(a \Rightarrow c), \forall a, b, c \in B$

Definition 2 (see [7]). Let $(B, \Rightarrow, 1)$ be an implication algebra. Then a nonempty subset $S$ of an implication algebra $B$ is called a subalgebra of $B$ if $a, b \in S$, then $a \Rightarrow b \in S$.

\section{Main Results}

Definition 3. An algebra $(H, \Rightarrow, 1)$ of type $(2,0)$ is said to be Hilbert implication algebra if it satisfies the following for all a, b.c $\in H$ :

$H_{1}: a \Rightarrow(b \Rightarrow a)=1$

$H_{2}:(a \Rightarrow(b \Rightarrow c) \Rightarrow(a \Rightarrow b) \Rightarrow(a \Rightarrow c)=1$

$H_{3}$ : If $a \Rightarrow b=b \Rightarrow a=1$, then $a=b$ 
Definition 4. Let $H$ be a Hilbert implication algebra and " $\leq$ " be a partially ordered relation defined by $a \leq b$ implies that $a \Rightarrow b=1$ for $a, b \in H$.

Example 1. Let $H=\{0, a, b, 1\}$ with $0 \leq a \leq b \leq 1$ define “ $\Rightarrow$ " by the following table:

$a \Rightarrow(b \Rightarrow a)=a \Rightarrow a=1$.

Hence, $H_{1}$ holds. $a \Rightarrow(b \Rightarrow 1)=a \Rightarrow 1=1$ and $(a \Rightarrow b)$ $\Rightarrow(a \Rightarrow 1)=1 \Rightarrow 1=1$.

Which imply $a \Rightarrow(b \Rightarrow 1) \Rightarrow(a \Rightarrow b) \Rightarrow(a \Rightarrow 1)=1$.

Hence, $H_{2}$ holds. $a \Rightarrow b=1$ and $b \Rightarrow a=a$. Implies a $6=b$.

Hence, $H_{3}$ holds. Hence, $(H, \Rightarrow, 1)$ is Hilbert implication algebra with respect to " $\leq$ ".

Definition 5. $H=(H, \Rightarrow, 1)$ is a groupoid if it satisfies the following axioms for all.

$$
\begin{aligned}
& a, b, c \in H: \\
& H_{1}:(a \Rightarrow b) \Rightarrow a=a \text { (contraction) } \\
& H_{2}:(a \Rightarrow b) \Rightarrow b=(b \Rightarrow a) \Rightarrow a \text { (quasicommutative) } \\
& H_{3}: a \Rightarrow(b \Rightarrow c)=b \Rightarrow(a \Rightarrow c) \text { (exchange rule) }
\end{aligned}
$$

Lemma 6. A groupoid is a Hilbert implication algebra.

Proof. Let $H=(H, \Rightarrow, 1)$ be a groupoid for $a, b, c \in H$. $a \Rightarrow(b \Rightarrow a)=b \Rightarrow(a \Rightarrow a)$ by $H_{3}=b \Rightarrow 1=1$.

Hence, $a \Rightarrow(b \Rightarrow a)=1$. Thus, $H_{1}$ holds. $[a \Rightarrow(b \Rightarrow c)] \Rightarrow[(a \Rightarrow b) \Rightarrow(a \Rightarrow c)]=[b \Rightarrow(a \Rightarrow c)] \Rightarrow$ $[(a \Rightarrow b) \Rightarrow(a \Rightarrow c)]$ by $H_{3} .=(a \Rightarrow b) \Rightarrow[(b \Rightarrow(a \Rightarrow c) \Rightarrow(a$ $\Rightarrow c)]=(a \Rightarrow b) \Rightarrow[b \Rightarrow((a \Rightarrow c) \Rightarrow(a \Rightarrow c)]=(a \Rightarrow b) \Rightarrow$ $(b \Rightarrow 1)$, since $(a \Rightarrow c) \Rightarrow(a \Rightarrow c)=1 .=(a \Rightarrow b) \Rightarrow 1=1$, since $(a \Rightarrow b) \Rightarrow(b \Rightarrow 1)=1$, and $b \Rightarrow 1=1$. Hence, $[a \Rightarrow(b$ $\Rightarrow c)] \Rightarrow[(a \Rightarrow b) \Rightarrow(a \Rightarrow c)]=1$.

Suppose $a, b \in H$ such that $a \Rightarrow b=1=b \Rightarrow a$. Assume $a$ $\leq b$. Then, $b \Rightarrow a=a$. But $b \Rightarrow a=1$. Hence, $a=1$. Again, assume $b \leq a$. Then, $a \Rightarrow b=b$. But $a \Rightarrow b=1$. Hence, $b=1$. Therefore, $a=b$. Thus, a groupoid is a Hilbert implication algebra.

Definition 7 . Let $(H, \Rightarrow, 1)$ be a Hilbert implication algebra. Then, for any $a, b \in H$, we have $a \Rightarrow b=\left\{\begin{array}{ll}1 & \text { if } a \leq b \\ b & \text { if } a>b\end{array}\right.$.

Lemma 8. Let $(H, \Rightarrow, 1)$ be a Hilbert implication algebra. Then, the following hold for all $a, b, 1 \in H$ :

(1) $a \Rightarrow a=1$

(2) $1 \Rightarrow a=a$

(3) $a \Rightarrow 1=1$

If $1 \Rightarrow a=1$, then $a=1$.

If $a \Rightarrow(b \Rightarrow a)=1$, then $a \leq b \Rightarrow a$.

$b \Rightarrow[(b \Rightarrow a) \Rightarrow a]=1$ if and only if $b \leq(b \Rightarrow a) \Rightarrow a$.

Proof.

(1) $a \Rightarrow a=1$, since $a \leq a$ by Definition 4 .
(2) Let $a \in H$, and 1 is largest element in $H$. Then, $1 \Rightarrow$ $a=1$ if $1 \leq a$ and $a$ if $a \leq 1$. But 1 is largest element in $H$ we have $a \leq 1$. Hence, $1 \Rightarrow a=a$.

(3) Let $a, 1 \in H$. Then, $a \Rightarrow 1=\left\{\begin{array}{ll}1 & \text { if } 1 \leq a \\ 1 & \text { if } a \leq 1\end{array}\right.$. But $a \leq 1$, for all $a \in H$

Hence, $a \Rightarrow 1=1$ for all $a \in H$.

(4) Let $a \in H$ and 1 is the largest element in $H$. Suppose $1 \Rightarrow a=1$. But $a \leq 1$. So that we have $1 \Rightarrow a=a$. Imply that $a=1$

(5) Let $a, b \in H$. Then $a \Rightarrow(b \Rightarrow a)=1$. By Definition 4, we have $a \Rightarrow(b \Rightarrow a)=\left\{\begin{array}{ll}1 & \text { if } a \leq b \\ b \Rightarrow a & \text { if } b \Rightarrow a \Rightarrow a\end{array}\right.$. But $a \Rightarrow(b \Rightarrow a)=1$

Hence, $a \leq b \Rightarrow a$.

Conversely suppose $\boldsymbol{a} \leq \boldsymbol{b} \Rightarrow \boldsymbol{a}$, for $\boldsymbol{a}, \boldsymbol{b} \in \boldsymbol{H} . \boldsymbol{a} \Rightarrow(b \Rightarrow a)$ $=\left\{\begin{array}{ll}1 & \text { if } a \leq b \Rightarrow a \\ b \Rightarrow a & \text { if } b \Rightarrow a \leq a\end{array}\right.$.

So that we have $\boldsymbol{a} \Rightarrow(\boldsymbol{b} \Rightarrow \boldsymbol{a})=\mathbf{1}$, since $\boldsymbol{a} \leq \boldsymbol{b} \Rightarrow \boldsymbol{a}$.

Let $\boldsymbol{a}, \boldsymbol{b} \in \boldsymbol{H}$, and let $\boldsymbol{b} \Rightarrow[(\boldsymbol{b} \Rightarrow \boldsymbol{a}) \Rightarrow \boldsymbol{a}]=\mathbf{1}$.

Now, $b \Rightarrow[(b \Rightarrow a) \Rightarrow a]=$

$\left\{\begin{array}{ll}1 & \text { if } b \leq(b \Rightarrow a) \Rightarrow a \\ (b \Rightarrow a) \Rightarrow a & \text { if }(b \Rightarrow a) \Rightarrow a \leq b\end{array}\right.$.

Imply that $\boldsymbol{b} \Rightarrow[(\boldsymbol{b} \Rightarrow \boldsymbol{a}) \Rightarrow \boldsymbol{a}]=\mathbf{1}$ by hypothesis.

Thus, $\boldsymbol{b} \leq(\boldsymbol{b} \Rightarrow \boldsymbol{a}) \Rightarrow \boldsymbol{a}$.

Conversely, let $\boldsymbol{a}, \boldsymbol{b} \in \boldsymbol{H}$, and $\boldsymbol{b} \leq(\boldsymbol{b} \Rightarrow \boldsymbol{a}) \Rightarrow \boldsymbol{a}$. Now $b \Rightarrow$

$[(b \Rightarrow a) \Rightarrow a]=\left\{\begin{array}{ll}1 & \text { if } b \leq(b \Rightarrow a) \Rightarrow a \\ (b \Rightarrow a) \Rightarrow a & \text { if }(b \Rightarrow a) \Rightarrow a \leq b\end{array}\right.$.

But $\boldsymbol{b} \leq(\boldsymbol{b} \Rightarrow \boldsymbol{a}) \Rightarrow \boldsymbol{a}$.

Therefore, $\boldsymbol{b} \Rightarrow[(\boldsymbol{b} \Rightarrow \boldsymbol{a}) \Rightarrow \boldsymbol{a}]=\mathbf{1}$.

Example 2. Let $H=\{a, b, 1\}$ with $a<b<1$. Then, $(H, \Rightarrow, 1)$ defined by the following table is not Hilbert implication algebra

Since $\boldsymbol{a} \Rightarrow(\boldsymbol{b} \Rightarrow \boldsymbol{a})=\boldsymbol{a} \Rightarrow \mathbf{1}=a \neq 1$. Hence, $\boldsymbol{H}_{\mathbf{1}}$ is not satisfied.

Lemma 9. Let $(\boldsymbol{H}, \Rightarrow, 1)$ be implication Hilbert algebra, and $\boldsymbol{a} \leq \boldsymbol{b} \Leftrightarrow \boldsymbol{a} \Rightarrow \boldsymbol{b}=1$. Then, $(\boldsymbol{H}, \leq)$ is a poset.

Proof.

(1) Let $a \in H$ and $a \leq a$. Imply that $a \Rightarrow a=1$. Hence, " $\leq$ " is reflexive

(2) Let $a, b \in H$, and $a \leq b$, and $b \leq a$. So that we have $a$ $\Rightarrow b=1$ and $b \Rightarrow a=1$ 
Imply $a \Rightarrow b=b \Rightarrow a$. Hence, $a=b$ by Definition 3 . Thus, " $\leq$ " is antisymmetric.

(3) Let $a, b, c \in H$ with $a \leq b$, and $b \leq c$. Then, $a \Rightarrow b=1$, and $b \Rightarrow c=1$

Now $a \leq b \leq c$ imply $a \leq c$. Imply that $a \Rightarrow c=1$ by Definition 4.

Therefore, $(\boldsymbol{H}, \leq)$ is a poset.

Example 3. Let $H=\{a, b, 1\}$ with $a<b<1$. Then, $(H, \leq)$ given by the following table is a poset.

Since

(1) $a \Rightarrow a=1, \quad b \Rightarrow b=1, \quad 1 \Rightarrow 1=1$. Hence, reflexive property holds.

(2) $a \Rightarrow b=1$, and $b \Rightarrow a=a \cdot a \Rightarrow b 6=b \Rightarrow a$. Imply that a $6=b$. Hence, antisymmetric property holds

(3) $a \Rightarrow b=1$, and $b \Rightarrow 1=1$. Imply that $a \Rightarrow 1=1$. Hence, the transitive property holds. Thus, $(H, \leq)$ is a poset

Theorem 10. Let $(\boldsymbol{H}, \Rightarrow, 1)$ be Hilbert implication algebra of type $(2,0)$. Then, $(\boldsymbol{a} \Rightarrow \boldsymbol{b}) \Rightarrow(1 \Rightarrow \boldsymbol{b})=\boldsymbol{a}$.

Proof. Let $\boldsymbol{a}, \boldsymbol{b}, \mathbf{1} \in \boldsymbol{H}$. Then, $\mathbf{1} \Rightarrow \boldsymbol{b}=\boldsymbol{b}$, since $\boldsymbol{b} \leq \mathbf{1}$. Now ( $\boldsymbol{a}$ $\Rightarrow \boldsymbol{b}) \Rightarrow(\mathbf{1} \Rightarrow \boldsymbol{b})=(\boldsymbol{a} \Rightarrow \boldsymbol{b}) \Rightarrow \boldsymbol{b}=(\boldsymbol{b} \Rightarrow \boldsymbol{a}) \Rightarrow \boldsymbol{a}=\boldsymbol{a}$. Hence, $(\boldsymbol{a} \Rightarrow \boldsymbol{b}) \Rightarrow(\mathbf{1} \Rightarrow \boldsymbol{b})=\boldsymbol{a}$.

Corollary 11. If $(\boldsymbol{H}, \Rightarrow, 1)$ is a Hilbert implication algebra, then $\boldsymbol{a} \Rightarrow \boldsymbol{c}=\boldsymbol{b} \Rightarrow \boldsymbol{c}$ implies that $\boldsymbol{a}=\boldsymbol{b}$ for any $\boldsymbol{a}, \boldsymbol{b}, \boldsymbol{c} \in \boldsymbol{H}$.

Lemma 12. Let $(\boldsymbol{H}, \Rightarrow, 1)$ be a Hilbert implication algebra. Then, for any $\boldsymbol{a}, \boldsymbol{b} \in \boldsymbol{H}$, the following hold: (1) $\boldsymbol{a} \Rightarrow \boldsymbol{b}=1$ implies $\boldsymbol{a}=\boldsymbol{b}$. (2) $1 \Rightarrow \boldsymbol{a}=1 \Rightarrow \boldsymbol{b}$ implies $\boldsymbol{a}=\boldsymbol{b}$. (3) $1 \Rightarrow(1 \Rightarrow$ a) $=\boldsymbol{a}$.

Proof.

(1) Let $\boldsymbol{a}, \boldsymbol{b} \in \boldsymbol{H}$, and $\boldsymbol{a} \Rightarrow \boldsymbol{b}=\mathbf{1}$. Then by Definition $4, a$ $\Rightarrow b=\left\{\begin{array}{ll}1 & \text { if } a \leq b \\ b & \text { if } b \leq a\end{array}\right.$. So that we have $\boldsymbol{a} \Rightarrow \boldsymbol{b}=\mathbf{1}$ if $\boldsymbol{b}$ $\geq \boldsymbol{a}$. Implies $\boldsymbol{a}=\boldsymbol{b}$ taking equality

(2) Let $\boldsymbol{a}, \boldsymbol{b}, \mathbf{1} \in \boldsymbol{H}$, and $\mathbf{1} \Rightarrow \boldsymbol{a}=\mathbf{1} \Rightarrow \boldsymbol{b}$. Now $\mathbf{1} \Rightarrow \boldsymbol{a}=\boldsymbol{a}$, since $\boldsymbol{a} \leq \mathbf{1}$, and $\mathbf{1} \Rightarrow \boldsymbol{b}=\boldsymbol{b}$, since $\boldsymbol{b} \leq \mathbf{1}$. Hence, $\boldsymbol{a}=\boldsymbol{b}$

(3) Let $\boldsymbol{a}, \mathbf{1} \in \boldsymbol{H}$, and let 1 be the largest element. $\mathbf{1} \Rightarrow(\mathbf{1}$ $\Rightarrow \boldsymbol{a})=\mathbf{1} \Rightarrow \boldsymbol{a}$, since $\boldsymbol{a} \leq \mathbf{1}$, and $\mathbf{1} \Rightarrow \boldsymbol{a}=\boldsymbol{a}$. Therefore, $\mathbf{1} \Rightarrow(\mathbf{1} \Rightarrow \boldsymbol{a})=\boldsymbol{a}$ for all $\boldsymbol{a} \in \boldsymbol{H}$

Theorem 13. If $(\boldsymbol{H}, \Rightarrow, 1)$ is a Hilbert implication algebra, then $(1 \Rightarrow \boldsymbol{b}) \Rightarrow(\boldsymbol{a} \Rightarrow \boldsymbol{b})=1$ for any $\boldsymbol{a}, \boldsymbol{b} \in \boldsymbol{H}$.
Definition 14. A Hilbert implication algebra $\boldsymbol{H}$ is said to be commutative if

$$
a \Rightarrow(b \Rightarrow \mathbf{1})=b \Rightarrow(a \Rightarrow \mathbf{1}) \text { for any } a, b \in H .
$$

Example 4. The table in Example 3 is a commutative Hilbert implication algebra.

Proposition 15. If $(\boldsymbol{H}, \Rightarrow, 1)$ is a commutative Hilbert implication algebra. Then

(1) $(\boldsymbol{a} \Rightarrow 1) \Rightarrow(1 \Rightarrow \boldsymbol{b})=1$

(2) $(1 \Rightarrow \boldsymbol{a}) \Rightarrow(1 \Rightarrow \boldsymbol{b})=\boldsymbol{a} \Rightarrow \boldsymbol{b}$

Proof.

(1) Let $\boldsymbol{a}, \boldsymbol{b} \in \boldsymbol{H}$. Then, $(\boldsymbol{a} \Rightarrow \mathbf{1}) \Rightarrow(b \Rightarrow \mathbf{1})=\mathbf{1} \Rightarrow \mathbf{1}=\mathbf{1}$, since $a \Rightarrow 1=1$, and $b \Rightarrow 1=1$

(2) Let $\boldsymbol{a}, \boldsymbol{b} \in \boldsymbol{H}$. Then, $(\mathbf{1} \Rightarrow \boldsymbol{a}) \Rightarrow(\mathbf{1} \Rightarrow \boldsymbol{b})=\boldsymbol{a} \Rightarrow \boldsymbol{b}$, since $\boldsymbol{a} \leq \mathbf{1}$ imply $\mathbf{1} \Rightarrow \boldsymbol{a}=\boldsymbol{a}$, and $\boldsymbol{b} \leq \mathbf{1}$ imply $\mathbf{1} \Rightarrow \boldsymbol{b}=\boldsymbol{b}$. Hence, $(\mathbf{1} \Rightarrow \boldsymbol{a}) \Rightarrow(\mathbf{1} \Rightarrow \boldsymbol{b})=\boldsymbol{a} \Rightarrow \boldsymbol{b}$ for any $\boldsymbol{a}, \boldsymbol{b} \in \boldsymbol{H}$.

Definition 16. Let $\mathrm{H}$ be a Hilbert implication algebra and a nonempty subset $I$ of $H$ is called an ideal of $H$ if it satisfies for all $a, b \in H$ :

(a) $\mathbf{0} \in \boldsymbol{I}$

(b) If $\boldsymbol{b} \in \boldsymbol{I}$ and $\boldsymbol{a} \Rightarrow \boldsymbol{b} \in \boldsymbol{I}$. Then $\boldsymbol{a} \in \boldsymbol{I}$

Definition 17. Let $\boldsymbol{H}$ be a Hilbert implication algebra and a nonempty subset $\boldsymbol{F}$ of $\boldsymbol{H}$ is called a filter of $\boldsymbol{H}$ if it satisfies the following for all $\boldsymbol{a}, \boldsymbol{b} \in \boldsymbol{H}$ : (1) $\mathbf{1} \in \boldsymbol{F}$. (2) If $\boldsymbol{a} \in \boldsymbol{F}$ and $\boldsymbol{a} \Rightarrow$ $\boldsymbol{b} \in \boldsymbol{F}$, then $\boldsymbol{b} \in \boldsymbol{F}$.

Theorem 18. If $(\boldsymbol{H}, \Rightarrow, 1)$ is a commutative Hilbert implication algebra, and

$$
(\boldsymbol{a} \Rightarrow(1 \Rightarrow \boldsymbol{b})=\boldsymbol{b} \Rightarrow(1 \Rightarrow \boldsymbol{a}) \text {, then } \boldsymbol{a} \Rightarrow(\boldsymbol{a} \Rightarrow \boldsymbol{b})=1 \text {. }
$$

Proof. Suppose $(\boldsymbol{H}, \Rightarrow, \mathbf{1})$ be a commutative Hilbert implication algebra and $\boldsymbol{a} \Rightarrow(\mathbf{1} \Rightarrow \boldsymbol{b})=\boldsymbol{b} \Rightarrow(\mathbf{1} \Rightarrow \boldsymbol{a})$, for any $\boldsymbol{a}, \boldsymbol{b} \in$ $\boldsymbol{H}$. Now $\boldsymbol{a} \Rightarrow(\boldsymbol{a} \Rightarrow \boldsymbol{b})=\boldsymbol{a} \Rightarrow(\boldsymbol{a} \Rightarrow(\mathbf{1} \Rightarrow \boldsymbol{b}))$, since $\mathbf{1} \Rightarrow \boldsymbol{b}=\boldsymbol{b}$ $=\boldsymbol{a} \Rightarrow(\boldsymbol{b} \Rightarrow(\mathbf{1} \Rightarrow \boldsymbol{a}))$ by hypothesis $=\boldsymbol{a} \Rightarrow(\boldsymbol{b} \Rightarrow \boldsymbol{a})$, since 1 $\Rightarrow \boldsymbol{a}=\boldsymbol{a} .=\mathbf{1}$ by definition of Hilbert implication algebra. Hence, $\boldsymbol{a} \Rightarrow(\boldsymbol{a} \Rightarrow \boldsymbol{b})=\mathbf{1}$.

Definition 19. An algebra $(\boldsymbol{G}, \Rightarrow, \mathbf{1})$ is said to be a generalized Hilbert implication algebra with a binary operation " $\Rightarrow$ " and constant 1 which satisfies the following axioms for all $\boldsymbol{a}, \boldsymbol{b}, \boldsymbol{c}$ $\in \mathbf{G}$ :

$$
\begin{aligned}
& \mathbf{G}_{1}: 1 \Rightarrow a=a \\
& \mathbf{G}_{2}: a \Rightarrow a=1 \\
& \mathbf{G}_{3}: c \Rightarrow(b \Rightarrow a)=b \Rightarrow(c \Rightarrow a) \\
& \mathbf{G}_{4}: c \Rightarrow(b \Rightarrow a)=(c \Rightarrow b) \Rightarrow(c \Rightarrow a), \text { for all } a, b, c \in G
\end{aligned}
$$


TABLE 1

\begin{tabular}{lllll}
\hline$\Rightarrow$ & 0 & $a$ & $b$ & 1 \\
0 & 1 & 1 & 1 & 1 \\
$a$ & 0 & 1 & 1 & 1 \\
$\boldsymbol{b}$ & 0 & $\boldsymbol{a}$ & 1 & 1 \\
1 & 0 & $\boldsymbol{a}$ & $\boldsymbol{b}$ & 1 \\
\hline
\end{tabular}

TABLE 2

\begin{tabular}{llll}
\hline$\Rightarrow$ & $\boldsymbol{a}$ & $\boldsymbol{b}$ & 1 \\
$\boldsymbol{a}$ & 1 & 1 & $\boldsymbol{a}$ \\
$\boldsymbol{b}$ & 1 & 1 & 1 \\
1 & $\boldsymbol{a}$ & $\boldsymbol{b}$ & 1 \\
\hline
\end{tabular}

Proposition 20. Every Hilbert implication algebra is a generalized Hilbert implication algebra. But the converse does not hold.

Proof. Suppose $(\boldsymbol{H}, \Rightarrow, \mathbf{1})$ be a Hibert implication algebra. Then, some axiom of generalized Hilbert implication algebra is satisfied by Lemma 8 and $\boldsymbol{H}_{\mathbf{3}}$.

What is remain to prove $\boldsymbol{c} \Rightarrow(\boldsymbol{b} \Rightarrow \boldsymbol{a})=(\boldsymbol{c} \Rightarrow \boldsymbol{b}) \Rightarrow(\boldsymbol{c} \Rightarrow$ $\boldsymbol{a})$, for any $\boldsymbol{a}, \boldsymbol{b}, \boldsymbol{c} \in \boldsymbol{G}$.

By $\boldsymbol{H}_{2}$ of definition of Hilbert implication algebra, we have

$\boldsymbol{c} \Rightarrow(\boldsymbol{b} \Rightarrow \boldsymbol{a}) \Rightarrow(\boldsymbol{c} \Rightarrow \boldsymbol{b}) \Rightarrow(\boldsymbol{c} \Rightarrow \boldsymbol{a})=\mathbf{1}$. Then, we have the following conditions:

(1) $c \Rightarrow(b \Rightarrow a)=(c \Rightarrow b) \Rightarrow(c \Rightarrow a)$

(2) $c \Rightarrow(b \Rightarrow a) \leq(c \Rightarrow b) \Rightarrow(c \Rightarrow a)$

(3) $(c \Rightarrow b) \Rightarrow(c \Rightarrow a)=1$

So that case one is straightforward. For case two it holds for equality. But case three does not lead to true conclusion.

Hence, $\boldsymbol{c} \Rightarrow(\boldsymbol{b} \Rightarrow \boldsymbol{a})=(\boldsymbol{c} \Rightarrow \boldsymbol{b}) \Rightarrow(\boldsymbol{c} \Rightarrow \boldsymbol{a})$. Thus, the forward condition holds.

To show the converse does not holds, consider the following example for $\boldsymbol{H}=\{\boldsymbol{a}, \boldsymbol{b}, \mathbf{1}\}$ and a binary operation defined by the table below:

For all $\boldsymbol{a}, \boldsymbol{b}, \mathbf{1} \in \boldsymbol{H}$, we have

(1) $1 \Rightarrow a=a, 1 \Rightarrow b=b$

(2) $a \Rightarrow a=1$, and $b \Rightarrow b=1$

(3) $1 \Rightarrow(a \Rightarrow b)=1 \Rightarrow 1=1$, and $a \Rightarrow(1 \Rightarrow b)=a \Rightarrow b$ $=1$. Hence, $1 \Rightarrow(a \Rightarrow b)=a \Rightarrow(1 \Rightarrow b)$

(4) $a \Rightarrow(a \Rightarrow b)=a \Rightarrow 1=1$, and $(a \Rightarrow a) \Rightarrow(a \Rightarrow b)=1$ $\Rightarrow 1=1$. Hence, $a \Rightarrow(a \Rightarrow b)=(a \Rightarrow a) \Rightarrow(a \Rightarrow b)$

Therefore, $(\boldsymbol{H}, \Rightarrow, \mathbf{1})$ is a generalized Hilbert implication algebra. Since $\boldsymbol{a} \Rightarrow \boldsymbol{b}=\mathbf{1}=\boldsymbol{b} \Rightarrow \boldsymbol{a}$. Hence, $(\boldsymbol{H}, \Rightarrow, \mathbf{1})$ is not a Hilbert implication algebra.
TABLE 3

\begin{tabular}{llll}
\hline$\Rightarrow$ & $\boldsymbol{a}$ & $\boldsymbol{b}$ & 1 \\
$\boldsymbol{a}$ & 1 & 1 & 1 \\
$\boldsymbol{b}$ & $\boldsymbol{a}$ & 1 & 1 \\
1 & $\boldsymbol{a}$ & $\boldsymbol{b}$ & 1 \\
\hline
\end{tabular}

TABLE 4

\begin{tabular}{llll}
\hline$\Rightarrow$ & $\boldsymbol{a}$ & $\boldsymbol{b}$ & 1 \\
$\boldsymbol{a}$ & 1 & 1 & 1 \\
$\boldsymbol{b}$ & 1 & 1 & 1 \\
1 & $\boldsymbol{a}$ & $\boldsymbol{b}$ & 1 \\
\hline
\end{tabular}

Lemma 21. In any generalized Hilbert implication algebra $(\boldsymbol{G}, \Rightarrow, 1)$, the following holds for all $\boldsymbol{a}, \boldsymbol{b}, \boldsymbol{c} \in \boldsymbol{G}$ :

(1) $(b \Rightarrow c) \Rightarrow[(c \Rightarrow a) \Rightarrow(b \Rightarrow a)]=1$

(2) $(c \Rightarrow a) \Rightarrow[(b \Rightarrow c) \Rightarrow(b \Rightarrow c)]=1$

(3) $a \Rightarrow(a \Rightarrow b)=a \Rightarrow b$

(4) $a \Rightarrow(b \Rightarrow a)=1$

(5) $[a \Rightarrow(b \Rightarrow c)] \Rightarrow[(a \Rightarrow b) \Rightarrow(a \Rightarrow c)]=1$

(6) $a \Rightarrow 1=1$

(7) $b \Rightarrow[(b \Rightarrow a) \Rightarrow a]=1$

Proof. Let $(\mathbf{G}, \Rightarrow, \mathbf{1})$ be a generalized Hilbert implication algebra (Tables 1-4).

(1) $(b \Rightarrow c) \Rightarrow[(c \Rightarrow a) \Rightarrow(b \Rightarrow a)]=(b \Rightarrow c) \Rightarrow[b \Rightarrow((c$ $\Rightarrow a) \Rightarrow a)]=(b \Rightarrow c) \Rightarrow[b \Rightarrow((c \Rightarrow a) \Rightarrow(a \Rightarrow a)]$ $=(b \Rightarrow c) \Rightarrow[b \Rightarrow((c \Rightarrow a) \Rightarrow 1)]=(b \Rightarrow c) \Rightarrow(b \Rightarrow$ 1) $=(b \Rightarrow c) \Rightarrow 1=1$

Hence, $(b \Rightarrow c) \Rightarrow[(c \Rightarrow a) \Rightarrow(b \Rightarrow a)]=1$.

(2) $(c \Rightarrow a) \Rightarrow[(b \Rightarrow c) \Rightarrow(b \Rightarrow c)=(c \Rightarrow a) \Rightarrow 1=1$, since $(b \Rightarrow c) \Rightarrow(b \Rightarrow c)=1$. Thus, $(c \Rightarrow a)[(b \Rightarrow c)$ $\Rightarrow(b \Rightarrow c)]=1$

(3) $a \Rightarrow(a \Rightarrow b)=(a \Rightarrow a) \Rightarrow(a \Rightarrow b)$ by definition of generalized Hilbert implication algebra. $=1 \Rightarrow(a \Rightarrow$ $b)=a \Rightarrow b$. Which imply that $a \Rightarrow(a \Rightarrow b)=a \Rightarrow b$

(4) $a \Rightarrow(b \Rightarrow a)=(a \Rightarrow b) \Rightarrow(a \Rightarrow a)$ by definition of generalized Hilbert implication algebra. $(a \Rightarrow b) \Rightarrow 1$ $=1$. Hence, $a \Rightarrow(b \Rightarrow a)=1$

(5) $[a \Rightarrow(b \Rightarrow c)] \Rightarrow[(a \Rightarrow b) \Rightarrow(a \Rightarrow c)]=[(a \Rightarrow b) \Rightarrow$ $(a \Rightarrow c)] \Rightarrow[(a \Rightarrow b) \Rightarrow(a \Rightarrow c)]=1$. So that we have $[a \Rightarrow(b \Rightarrow c)] \Rightarrow[(a \Rightarrow b) \Rightarrow(a \Rightarrow c)]=1$ 
(6) $a \Rightarrow 1=a \Rightarrow(a \Rightarrow a)$, since $a \Rightarrow a=1 .=(a \Rightarrow a) \Rightarrow$ $(a \Rightarrow a)$ by definition of generalized Hilbert implication algebra. $=1 \Rightarrow 1=1$. Therefore, $a \Rightarrow 1=1$

(7) $b \Rightarrow[(b \Rightarrow a) \Rightarrow a]=(b \Rightarrow(b \Rightarrow a)) \Rightarrow(b \Rightarrow a) \Rightarrow=$ $[(b \Rightarrow b) \Rightarrow(b \Rightarrow a)] \Rightarrow(b \Rightarrow a)=[1 \Rightarrow(b \Rightarrow a)] \Rightarrow$ $(b \Rightarrow a)=(b \Rightarrow a) \Rightarrow(b \Rightarrow a)=1$. It follows that $b \Rightarrow[(b \Rightarrow a) \Rightarrow a]=1$

\section{Conclusion}

In this paper, the idea of Hilbert implication algebra and Generalized Hilbert implication algebra is introduced. Some of the properties of Hilbert implication algebra are investigated. Congruence relations on Hilbert implication algebra and some other properties will be considered as future research work.

\section{Data Availability}

As you know, this manuscript is abstract algebra part, the idea is taken from the reference material listed in the manuscript, I need this material to be published as open access research paper to be accessible to researchers, and I am doing this research to contribute my idea to the theory of Mathematics.

\section{Conflicts of Interest}

The author declares that he has no conflicts of interest.

\section{Acknowledgments}

The author of this paper would like to thank the referees for their genuine comment for the improvement of the manuscript.

\section{References}

[1] E. H. Roh, S. Y. Kim, Y. Xu, and Y. B. Jun, "Some operations in lattice implication algebras," International Journal of Mathematics and Mathematical Sciences, vol. 27, no. 1, p. 52, 2001.

[2] R. Halaš, "Remarks on commutative Hilbert algebras," Mathematica Bohemica, vol. 127, no. 4, pp. 525-529, 2002.

[3] I. Chajda, "Implication algebras," Discussion Mathematica General Algebra and Application, vol. 26, no. 2, pp. 141-153, 2006.

[4] R. A. Borzooei and J. Shohani, "On generalized Hilbert algebras," Italian Journal of Pure and Applied Mathematics, vol. 29, pp. 71-80, 2012.

[5] B. Assaye, M. Alemneh, and G. Tefera, "B-Almost distributive fuzzy lattice," Bulletin of the Section of Logic, vol. 47, no. 3, pp. 171-185, 2018.

[6] G. T. Dejen, "Pseudo-supplemented almost distributive fuzzy lattices," Journal of Applied Mathematics and Computation, vol. 5, no. 2, pp. 119-123, 2021.

[7] T. Gerima, Y. Endris, and G. Fasil, "Ideals and filters on implication algebras," Advances in Mathematics: Scientific Journals, vol. 10, no. 3, pp. 1167-1174, 2020. 